\title{
Tracking curvaton(s)?
}

\author{
Massimo Giovannini* \\ Theoretical Physics Division, CERN, CH-1211 Geneva 23, Switzerland
}

(Received 1 October 2003; published 9 April 2004)

\begin{abstract}
The ratio of the curvaton energy density to that of the dominant component of the background sources may be constant during a significant period in the evolution of the Universe. The possibility of having tracking curvatons, whose decay occurs prior to the nucleosynthesis epoch, is studied. It is argued that the tracking curvaton dynamics is disfavored since the value of the curvature perturbations prior to curvaton decay is smaller than the value required by observations. It is also argued, in a related context, that the minimal inflationary curvature scale compatible with the curvaton paradigm may be lowered in the case of low-scale quintessential inflation.
\end{abstract}

DOI: 10.1103/PhysRevD.69.083509

PACS number(s): 98.80.Cq, 98.70.Vc

\section{INTRODUCTION}

The isocurvature fluctuations generated by a field $\epsilon$ that is light during the inflationary phase and later on decays, can efficiently produce adiabatic curvature perturbations. This idea has recently been discussed in different frameworks, ranging from the context of conventional inflationary models $[1,2]$ to the case of pre-big-bang models $[3,5,4]$. It is appropriate to recall that an earlier version of this proposal was discussed in [6], not with the specific purpose of converting isocurvature into adiabatic modes, but rather with the hope of providing physical initial conditions for the baryon isocurvature perturbations.

A common feature of various scenarios [7-16] is that the energy density of the homogeneous component of this light curvaton field decreases more slowly than the energy density of the background geometry. In the simplest realization, the Universe suddenly becomes dominated by radiation, as soon as inflation ends. During the radiation epoch, the homogeneous component of the curvaton field is roughly constant down to the moment when the Hubble parameter is comparable with the curvaton mass, i.e. $H \sim m$. The energy density of the oscillating curvaton field then decreases as $a^{-3}$, where $a$ is the scale factor of a conformally flat FriedmannRobertson-Walker (FRW) Universe. Since the energy density of the background radiation scales as $a^{-4}$, the ratio

$$
r(a)=\frac{\rho_{\epsilon}(a)}{\rho_{\mathrm{r}}(a)}
$$

will increase as $a$. Both a different post-inflationary history and a different potential will produce deviations from this scaling law. In particular, if the potential is not exactly quadratic (or if the coupling of the curvaton to the geometry is not exactly minimal) [13] $r(a)$ may decrease. If the postinflationary phase is not immediately dominated by radiation, $r(a)$ may increase even faster than $a$. If, after the end of inflation the inflaton field turns into a quintessence-like field [17] (see also $[15,18]$ ), the background energy density will

\footnotetext{
*Electronic address: massimo.giovannini@cern.ch
}

be dominated by the kinetic term of the quintessence field. In this case $r(\eta)$ increases as $a^{3}$ [9].

It is not impossible that the ratio $r(a)$ stays constant during a significant period in the post-inflationary evolution. This subject will be explored in the present investigation. It will be argued that this type of "tracking" phase is disfavored because the initial isocurvature mode will (rather generically) turn into an adiabatic mode of much smaller amplitude, making the whole scenario ineffective in correctly reproducing the amplitude of the large-scale fluctuations.

One of the reasons to speculate on the possibility of tracking curvatons is that one would like to allow for models where the curvature scale, at the end of inflation, is much smaller than the value required, for instance, in single field inflationary models, where the curvature fluctuations are directly amplified during the inflationary phase. It will be shown that if the inflationary phase is not followed suddenly by radiation but rather by a kinetic phase (as in the case of quintessential inflation) the minimal allowed curvature scale is a bit smaller than in the standard case of sudden radiation domination.

The present paper is organized as follows. In Sec. II the basic problem will be formulated. In Sec. III it will be shown that if the curvaton field tracks the evolution of the dominant component of the background the resulting adiabatic mode is smaller then the initial isocurvature mode. In Sec. IV the lower bound on the inflationary curvature scale will be discussed. Finally, Sec. V contains some concluding remarks.

\section{FORMULATION OF THE PROBLEM}

For illustrative purposes it is useful to consider the case where the inflationary phase is suddenly replaced by a radiation-dominated phase. During inflation, the curvaton field $\epsilon$ should be nearly massless and displaced from the minimum of its potential $W(\epsilon)$ :

$$
\frac{\partial^{2} W}{\partial \epsilon^{2}} \ll H_{\mathrm{i}}^{2}, \quad\left|\epsilon_{\mathrm{i}}-\epsilon_{0}\right|>H_{\mathrm{i}}
$$

where the subscript denotes the moment when the cosmologically interesting scales left the horizon during inflation. 
Under the assumption that the Universe is dominated by radiation, the evolution equations for the background fields can be written ${ }^{1}$

$$
\begin{gathered}
M_{\mathrm{P}}^{2} \mathcal{H}^{2}=\frac{a^{2}}{3}\left(\rho_{\epsilon}+\rho_{\mathrm{r}}\right), \\
M_{\mathrm{P}}^{2} \mathcal{H}^{\prime}=-\frac{1}{3}\left[a^{2} \rho_{\mathrm{r}}+\epsilon^{\prime 2}-W a^{2}\right], \\
\epsilon^{\prime \prime}+2 \mathcal{H} \epsilon^{\prime}+\frac{\partial W}{\partial \epsilon} a^{2}=0, \\
\rho_{\mathrm{r}}^{\prime}+4 \mathcal{H} \rho_{\mathrm{r}}=0,
\end{gathered}
$$

where it has been assumed that the curvaton is minimally coupled to the background geometry. This assumption may be relaxed by requiring, for instance, that the coupling be non-minimal. This would imply the addition, in Eq. (2.4), of a term going like $c R \epsilon$, where $R \propto H^{2}$ is the Ricci scalar of a radiation-dominated FRW background. The addition of such a term is expected to lead to a decrease of $r(a)$ [13] even prior to the true oscillatory phase taking place when $H$ $\sim m$. For the purposes of the present section, it is useful to parametrize the evolution of $\epsilon$ in terms of $r=\rho_{\epsilon} / \rho_{\mathrm{r}}$. In this case

$$
\begin{aligned}
& \epsilon^{\prime 2}=-M_{\mathrm{P}}^{2} \mathcal{H} a^{4}\left(\frac{r}{a^{4}}\right)^{\prime}, \\
& \frac{\partial W}{\partial \epsilon} a^{2}=-\frac{1}{a^{2}}\left(a^{2} \epsilon^{\prime}\right)^{\prime},
\end{aligned}
$$

where the first equation has been obtained by taking the derivative of the definition of $r$ and by subsequent use of Eq. (2.4).

Provided $r\left(\eta_{\mathrm{i}}\right) \ll 1$, three possible physical situations may arise. If $r^{\prime}>0$, the curvaton field will become, at some point, dominant over the background density. If $r^{\prime}<0$, the curvaton field will never dominate over the background geometry. The third possibility is to have $r^{\prime} \simeq 0$, which is the case to be discussed in the following. Even if examples will be provided in the framework of specific potentials, it is better, at this stage, to think of the potential as a function of the ratio $r$, as suggested by Eqs. (2.6). The perturbation equations relevant in order to study the dynamical evolution of the field $\epsilon$ are obtained by linearizing the evolution equations to first

\footnotetext{
${ }^{1}$ Units $M_{\mathrm{P}}=(8 \pi G)^{-1 / 2}$ will be adopted. The variable $\eta$ denotes the conformal time coordinate while $t$ denotes the cosmic time. Derivatives with respect to either conformal or cosmic time will be denoted, respectively, by a prime and by an overdot. Finally, the expansion rate, in the conformal and cosmic time coordinates will be denoted by $\mathcal{H}=a^{\prime} / a$ and by $H=\dot{a} / a$.
}

order in the amplitude of the metric fluctuations. In general terms, the scalar fluctuations of a conformally flat metric of the type

$$
\bar{g}_{\mu \nu}=a^{2}(\eta) \eta_{\mu \nu}
$$

can be parametrized as

$$
\begin{aligned}
\delta^{(1)} g_{00} & =2 a^{2}(\eta) \phi, \\
\delta^{(1)} g_{i j} & =2 a^{2}(\eta)\left(\psi \delta_{i j}-\partial_{i} \partial_{j} E\right), \\
\delta^{(1)} g_{0 i} & =-a^{2}(\eta) \partial_{i} B,
\end{aligned}
$$

where $\phi, \psi, B$ and $E$ are the four scalar degrees of freedom of the perturbed metric; $\delta^{(1)}$ denotes the first order fluctuation of the corresponding quantity. Since the infinitesimal coordinate transformations preserving the scalar nature of the fluctuation have two parameters, out of the four scalar fluctuations defined in Eq. (2.8), two gauge-invariant Bardeen potentials can be defined, i.e.

$$
\begin{aligned}
& \Phi=\phi+\mathcal{H}\left(B-E^{\prime}\right)+\left(B-E^{\prime}\right)^{\prime}, \\
& \Psi=\psi-\mathcal{H}\left(B-E^{\prime}\right) .
\end{aligned}
$$

The first order perturbation of the Einstein equations, of the curvaton equations and of the covariant conservation of the energy-momentum tensor of the fluid can be written, respectively, as

$$
\delta^{(1)} R_{\mu}^{\nu}-\frac{1}{2} \delta_{\mu}^{\nu} \delta^{(1)} R=\frac{1}{M_{\mathrm{P}}^{2}} \delta^{(1)} T_{\mu}^{\nu}+\frac{1}{M_{\mathrm{P}}^{2}} \delta^{(1)} \mathcal{T}_{\mu}^{\nu}
$$

$$
\begin{aligned}
\bar{g}^{\mu \nu}\left[\partial_{\mu} \partial_{\nu} \delta^{(1)} \epsilon-\delta^{(1)} \Gamma_{\mu \nu}^{\sigma} \partial_{\sigma} \epsilon+\bar{\Gamma}_{\mu \nu}^{\sigma} \partial_{\sigma} \delta^{(1)} \epsilon\right] \\
+\delta^{(1)} g^{\mu \nu}\left(\partial_{\mu} \partial_{\nu}-\bar{\Gamma}_{\mu \nu}^{\sigma} \partial_{\sigma} \epsilon\right)+\frac{\partial^{2} W}{\partial \epsilon^{2}} \delta^{(1)} \epsilon=0,
\end{aligned}
$$

$$
\begin{aligned}
& \partial_{\mu} \delta^{(1)} \mathcal{T}_{\nu}^{\mu}-\delta^{(1)} \Gamma_{\mu \nu}^{\alpha} \overline{\mathcal{T}}_{\alpha}^{\mu}-\bar{\Gamma}_{\mu \nu}^{\alpha} \delta^{(1)} \mathcal{T}_{\alpha}^{\mu}+\delta^{(1)} \Gamma_{\mu \alpha}^{\mu} \overline{\mathcal{T}}_{\nu}^{\alpha} \\
& +\Gamma_{\mu \alpha}^{\mu} \delta^{(1)} \mathcal{T}_{\nu}^{\alpha}=0
\end{aligned}
$$

where $T_{\mu}^{\nu}$ is the energy-momentum tensor of the curvaton and $\mathcal{T}_{\mu}^{\nu}$ is the energy-momentum tensor of the fluid sources. In terms of the two gauge-invariant Bardeen potentials defined in Eq. (2.9) the gauge-invariant fluctuations of the energy-momentum tensors can be written as 


$$
\begin{aligned}
& \delta^{(\text {gi })} T_{i}^{j}=\frac{1}{a^{2}}\left[\epsilon^{\prime 2} \Phi-\epsilon^{\prime} \chi^{\prime}+\frac{\partial W}{\partial \epsilon} a^{2} \chi\right], \\
& \delta^{(\text {gi })} T_{0}^{0}=\frac{1}{a^{2}}\left[-\epsilon^{\prime 2} \Phi+\epsilon^{\prime} \chi^{\prime}+\frac{\partial W}{\partial \epsilon} a^{2} \chi\right], \\
& \delta^{\text {(gi) }} T_{i}^{0}=\frac{1}{a^{2}} \epsilon^{\prime} \chi, \\
& \delta^{(\text {gi })} \mathcal{T}_{i}^{j}=-\frac{1}{3} \delta \rho_{\mathrm{r}} \delta_{i}^{j} \\
& \delta^{\text {(gi) } \mathcal{T}_{0}^{0}}=\delta \rho_{\mathrm{r}} \\
& \delta^{\text {(gi) } \mathcal{T}_{i}^{0}}=\frac{4}{3} \rho_{\mathrm{r}} \partial_{i} u,
\end{aligned}
$$

where

$$
\chi=\delta^{(1)} \epsilon+\epsilon^{\prime}\left(B-E^{\prime}\right)
$$

is the gauge-invariant fluctuation of the curvaton and

$$
\begin{gathered}
\delta \rho_{\mathrm{r}}=\delta^{(1)} \rho_{\mathrm{r}}+\rho_{\mathrm{r}}^{\prime}\left(B-E^{\prime}\right), \\
u=\delta^{(1)} u+\left(B-E^{\prime}\right)
\end{gathered}
$$

are, respectively, the gauge-invariant fluctuations of the fluid energy and of the velocity potential. Inserting Eqs. (2.13)(2.15) into the $(i, j)$ component of Eqs. (2.10) the following relation is obtained:

$$
\partial_{i} \partial^{j}(\Phi-\Psi)=0
$$

implying $\Phi=\Psi$. From the $(0,0),(i=j)$ and $(0, i)$ components of Eq. (2.10) and using the background equations (2.2)-(2.5), the following equations can be, respectively, obtained:

$$
\begin{gathered}
\nabla^{2} \Phi-3 \mathcal{H}\left(\mathcal{H} \Phi+\Phi^{\prime}\right) \\
=\frac{a^{2}}{2 M_{\mathrm{P}}^{2}} \rho_{\mathrm{r}} \delta_{\mathrm{r}}+\frac{1}{2 M_{\mathrm{P}}^{2}}\left[-\Phi \epsilon^{\prime 2}+\epsilon^{\prime} \chi^{\prime}+\frac{\partial W}{\partial \epsilon} a^{2} \chi\right], \\
\Phi^{\prime \prime}+3 \mathcal{H} \Phi^{\prime}+\left(\mathcal{H}^{2}+2 \mathcal{H}^{\prime}\right) \Phi \\
=\frac{a^{2}}{6 M_{\mathrm{P}}^{2}} \rho_{\mathrm{r}} \delta_{\mathrm{r}}-\frac{1}{2 M_{\mathrm{P}}^{2}}\left[\Phi \epsilon^{\prime 2}-\epsilon^{\prime} \chi^{\prime}+\frac{\partial W}{\partial \epsilon} a^{2} \chi\right] \\
\mathcal{H} \Phi+\Phi^{\prime}=\frac{\epsilon^{\prime}}{2 M_{\mathrm{P}}^{2}} \chi+\frac{2}{3 M_{\mathrm{P}}^{2}} u a^{2} \rho_{\mathrm{r}}
\end{gathered}
$$

where $\delta_{\mathrm{r}}=\delta \rho_{\mathrm{r}} / \rho_{\mathrm{r}}$ and where Eq. (2.16) has been used. Finally, using Eqs. (2.13)-(2.15) and (2.16) into Eqs. (2.11) and (2.12) the explicit form of the remaining equations will be

$$
\begin{gathered}
\chi^{\prime \prime}+2 \mathcal{H} \chi^{\prime}-\nabla^{2} \chi+\frac{\partial^{2} W}{\partial \epsilon^{2}} a^{2} \chi-4 \epsilon^{\prime} \Phi^{\prime}+2 \frac{\partial W}{\partial \epsilon} a^{2} \Phi=0 \\
\delta_{\mathrm{r}}^{\prime}-4 \Phi^{\prime}-\frac{4}{3} \nabla^{2} u=0 \\
u^{\prime}-\frac{1}{4} \delta_{\mathrm{r}}-\Phi=0
\end{gathered}
$$

where Eq. (2.20) is the perturbed curvaton equation; Eqs. (2.21) and (2.22) correspond to the (0) and $(i)$ components of Eq. (2.12).

Introducing the useful notation $x=\ln \left(\eta / \eta_{\mathrm{i}}\right)$, Eqs. (2.17), (2.18) and (2.20) can be written, in Fourier space,

$$
\begin{aligned}
\frac{d \Phi_{k}}{d x}+ & \Phi_{k} \\
= & -\frac{\delta_{\mathrm{r}}(k)}{2}-\frac{1}{6 M_{\mathrm{P}}^{2}}\left[-\Phi_{k}\left(\frac{d \epsilon}{d x}\right)^{2}+\frac{d \epsilon}{d x} \frac{d \chi_{k}}{d x}\right. \\
& \left.+e^{4 x} \eta_{\mathrm{i}}^{2} \frac{\partial W}{\partial \epsilon} \chi_{k}\right],
\end{aligned}
$$

$$
\begin{aligned}
\frac{d^{2} \Phi_{k}}{d x^{2}} & +2 \frac{d \Phi_{k}}{d x}-\Phi_{k} \\
= & \frac{\delta_{\mathrm{r}}(k)}{2}-\frac{1}{2 M_{\mathrm{P}}^{2}}\left[\Phi_{k}\left(\frac{d \epsilon}{d x}\right)^{2}-\frac{d \epsilon}{d x} \frac{d \chi_{k}}{d x}+e^{4 x} \eta_{i}^{2} \frac{\partial W}{\partial \epsilon} \chi_{k}\right],
\end{aligned}
$$

$\frac{d^{2} \chi_{k}}{d x^{2}}+\frac{d \chi_{k}}{d x}+e^{4 x} \eta_{\mathrm{i}}^{2} \frac{\partial^{2} W}{\partial \epsilon^{2}} \chi_{k}-4 \frac{d \epsilon}{d x} \frac{d \Phi_{k}}{d x}+2 \frac{\partial W}{\partial \epsilon} e^{4 x} \eta_{\mathrm{i}}^{2} \Phi_{k}=0$.

Combining Eqs. (2.23) and (2.24),

$$
\begin{aligned}
& \frac{d^{2} \Phi_{k}}{d x^{2}}+3 \frac{d \Phi_{k}}{d x}+\frac{1}{3 M_{\mathrm{P}}^{2}}\left[\Phi\left(\frac{d \epsilon}{d x}\right)^{2}-\frac{d \epsilon}{d x} \frac{d \chi_{k}}{d x}+2 e^{4 x} \eta_{\mathrm{i}}^{2} \frac{\partial W}{\partial \epsilon} \chi_{k}\right] \\
& =0 .
\end{aligned}
$$

Imposing isocurvature initial conditions right at the onset of the radiation dominated epoch implies, for long-wavelength modes:

$$
\Phi_{k}\left(\eta_{\mathrm{i}}\right)=0, \quad \Phi_{k}^{\prime}\left(\eta_{\mathrm{i}}\right)=0, \quad \chi_{k}\left(\eta_{\mathrm{i}}\right)=\chi_{k}^{(\mathrm{i})}, \quad \chi_{k}^{\prime}\left(\eta_{\mathrm{i}}\right)=0 .
$$

In terms of $\mathcal{R}_{k}$, the gauge-invariant spatial curvature perturbations, 


$$
\mathcal{R}_{k}=-\left(\Phi_{k}+\mathcal{H} \frac{\mathcal{H} \Phi_{k}+\Phi_{k}^{\prime}}{\mathcal{H}^{2}-\mathcal{H}^{\prime}}\right),
$$

the initial conditions given in Eq. (2.27) imply $\mathcal{R}_{k}\left(\eta_{\mathrm{i}}\right)=0$. From the Hamiltonian constraint, recalling Eq. (2.27), the relation between the initial density contrast and the initial curvaton fluctuation can be obtained

$$
\delta_{\mathrm{r}}^{(\mathrm{i})}(k)=-\left[\frac{\partial W}{\partial \epsilon}\right]_{\eta_{\mathrm{i}}} \frac{\chi_{k}^{(\mathrm{i})}}{\rho_{r}^{(\mathrm{i})}},
$$

where $\delta_{\mathrm{r}}^{(\mathrm{i})}(k)=\delta_{\mathrm{r}}\left(k, \eta_{\mathrm{i}}\right)$. Using the observation that, from Eq. (2.21), the quantity $\delta_{\mathrm{r}}(k)-4 \Phi_{k}$ is conserved in the longwavelength limit the initial value of the isocurvature mode can be related to the final values both of the Bardeen potential and of the curvaton fluctuation. On this basis, useful analytical relations will be derived and later compared with the numerical solutions. From Eq. (2.29) and taking into account Eq. (2.27), the Hamiltonian constraint of Eq. (2.23) leads to the relation

$$
\delta_{\mathrm{r}}^{(\mathrm{f})}(k)=4 \Phi_{k}^{(\mathrm{f})}-\left[\frac{\partial W}{\partial \epsilon}\right]_{\eta_{\mathrm{i}}} \frac{\chi_{k}^{(\mathrm{i})}}{\rho_{r}^{(\mathrm{i})}},
$$

where the superscript " $f$ " denotes the final (constant) value of the corresponding quantity. In the case $r^{\prime} \simeq 0$, from Eq. (2.6)

$$
\epsilon^{\prime} \simeq 2 \mathcal{H} M_{\mathrm{P}} \sqrt{r} .
$$

Then Eqs. (2.23)-(2.25) admit a solution with constant mode. Equation (2.25) and the combination of Eqs. (2.23) with (2.30) lead, respectively, to the following two relations:

$$
\begin{gathered}
\frac{\partial^{2} W}{\partial \epsilon^{2}} \chi_{k}^{(\mathrm{f})}+2 \frac{\partial W}{\partial \epsilon} \Phi_{k}^{(\mathrm{f})}=0, \\
\Phi_{k}^{(\mathrm{f})}=\frac{1}{6}\left(\frac{\partial W}{\partial \epsilon}\right) \frac{\chi_{k}^{(\mathrm{i})}}{\eta_{\mathrm{i}}},
\end{gathered}
$$

which allow, in turn, $\chi_{k}^{(\mathrm{f})}$ to be determined in terms of $\chi_{k}^{(\mathrm{i})}$, namely,

$$
\left(\frac{\partial^{2} W}{\partial \epsilon^{2}}\right)_{\eta_{\mathrm{f}}} \chi_{k}^{(\mathrm{f})}=-\frac{1}{3}\left(\frac{\partial W}{\partial \epsilon}\right)_{\eta_{\mathrm{i}}}\left(\frac{\partial W}{\partial \epsilon}\right) \underset{\eta_{\mathrm{f}}}{\frac{\chi_{k}^{(\mathrm{i})}}{\rho_{r}^{(\mathrm{i})}}} .
$$

Defining now the constant value of $r$ as $r_{\mathrm{c}}$, the previous results imply

$$
\Phi_{k}^{(\mathrm{f})} \simeq-\frac{\sqrt{r_{\mathrm{c}}}}{9} \widetilde{\chi}_{k}^{(\mathrm{i})}+\mathcal{O}\left(r_{\mathrm{c}}\right)
$$

where $\tilde{\chi}_{k}=\chi_{k} / M_{\mathrm{P}}$. From Eq. (2.35) it also follows, using Eq. (2.32) together with Eq. (2.6)

$$
\chi_{k}^{(\mathrm{f})} \simeq-\frac{2 r_{\mathrm{c}}}{9} \chi_{k}^{(\mathrm{i})}
$$

Equations (2.35) and (2.36) imply that $\Phi_{k}^{(\mathrm{f})}$ is always smaller (by a factor $\sqrt{r_{c}} \ll 1$ ) than the value of the curvaton fluctuations at the end of inflation. Hence, even assuming that the curvaton fluctuations are amplified with flat spectrum, i.e. $\chi_{k}^{(\mathrm{i})} \sim H_{\mathrm{i}} /(2 \pi)$, the final value of the produced adiabatic mode $\Phi_{k}^{(\mathrm{f})}$ will always be phenomenologically negligible. The value of $\chi_{k}^{(\mathrm{f})}$ will be even smaller. In the second place the above equations are derived assuming that, in the asymptotic regime, the following relation holds:

$$
\left.\left.\frac{1}{\dot{\epsilon}^{2}}\left(\frac{\partial W}{\partial \epsilon}\right)^{2}\right|_{\eta_{\mathrm{f}}} \simeq \frac{1}{4}\left(\frac{\partial^{2} W}{\partial \epsilon^{2}}\right)\right|_{\eta_{\mathrm{f}}} .
$$

The relation (2.37) holds exactly in the case of exponential potentials of the type $W(\epsilon)=W_{0} e^{-\epsilon / \Lambda}$. In this case the solution for $\epsilon$ is obtained by solving Eq. (2.4) in a radiationdominated background, with the result that

$$
\epsilon(\eta)=\epsilon_{\mathrm{i}}+\epsilon_{1} \ln \left(\eta / \eta_{\mathrm{i}}\right)
$$

where $\epsilon_{1}=4 \Lambda$ and $r_{c}=4\left(\Lambda / M_{\mathrm{P}}\right)^{2}$. This remark already rules out a curvaton potential, which is purely exponential down to the moment of curvaton decay. In this case Eqs. (2.35) and (2.36) imply the smallness of the obtained curvature perturbations. However, it is also suggestive to think of the case where the curvaton potential is not purely exponential $[19,20]$. The question would be, in this case, if the new features of the potential allow a radically different dynamics of the fluctuations.

\section{EXPLICIT EXAMPLES}

In order to discuss a physically realistic situation, consider as an example the following potential

$$
W(\epsilon)=W_{0}[\cosh (\epsilon / \Lambda)-1]^{\alpha},
$$

which has been studied, for instance, in the context of quintessence models [21]. In spite of this formal analogy, in the present case, the field $\epsilon$ will decay prior to big-bang nucleosynthesis and it will not act as a quintessence field. With this caveat, also other quintessential potentials (see, for instance, [22] for a comprehensive review) may be used, in the present context, to construct physical models. The class of potentials (3.1) has the property that when $|\epsilon / \Lambda| \gg 1$ the potential admits solutions where the ratio between the curvaton energy density and the radiation energy density is, momentarily, constant. On the other hand, when $|\epsilon / \Lambda| \geqslant 1$ the curvaton oscillates. Consider, for concreteness, the simplest case, namely,

$$
W(\epsilon)=M^{4}[\cosh (\epsilon / \Lambda)-1],
$$

where the oscillations are quadratic since, for $|\epsilon / \Lambda| \ll 1$, 


$$
W(\epsilon) \simeq \frac{M^{4}}{2 \Lambda^{2}} \epsilon^{2} .
$$

Using the notation

$$
\tilde{\epsilon}=\frac{\epsilon}{M_{\mathrm{P}}}, \quad \mu=\frac{\Lambda}{M_{\mathrm{P}}}, \quad \nu=\frac{M}{M_{\mathrm{P}}}, \quad \xi=\frac{H_{\mathrm{i}}}{M_{\mathrm{P}}},
$$

the evolution of the curvaton is determined by the following equation:

$$
\frac{d^{2} \tilde{\boldsymbol{\epsilon}}}{d x^{2}}+\frac{d \tilde{\boldsymbol{\epsilon}}}{d x}+e^{4 x} \frac{\nu^{4}}{\mu \xi^{2}} \sinh (\tilde{\boldsymbol{\epsilon}} / \mu)=0 .
$$

The constraints of Eq. (2.1) imply, in the present case,

$$
\frac{M^{4}}{\Lambda^{2}} e^{-\epsilon_{\mathrm{i}} / \Lambda} \ll H_{\mathrm{i}}^{2}, \quad\left|\epsilon_{\mathrm{i}}\right|>\Lambda, \quad\left|\epsilon_{\mathrm{i}}\right| \gg H_{\mathrm{i}} .
$$

The ratio between the curvaton energy density and the radiation energy density can be expressed as

$$
r(x)=\frac{1}{6}\left(\frac{d \tilde{\boldsymbol{\epsilon}}}{d x}\right)^{2}+\frac{\nu^{4}}{3 \xi^{2}} e^{4 x}[\cosh (\tilde{\boldsymbol{\epsilon}} / \mu)-1] .
$$

The initial data [obeying the conditions (3.6)] for the evolution of the homogeneous component of the curvaton field are set during the inflationary epoch in such a way that $\left|\tilde{\epsilon}_{\mathrm{i}}\right|$ $>\mu$. Since the potential is essentially exponential, in this regime the field will be swiftly attracted towards the tracking solution, where the critical fraction of curvaton's energy density will be constant. In this regime the solution can be approximated by

$$
\tilde{\epsilon}=-\tilde{\epsilon}_{0}+4 \mu x, \quad \tilde{\epsilon}_{0}=\mu \ln \left(\frac{8 \mu^{2} \xi^{2}}{\nu^{4}}\right) .
$$

When $H^{2} \sim M^{4} / \Lambda^{2} \sim M^{2} \nu^{2} / \mu^{2}$, i.e.

$$
x_{\mathrm{m}}=\frac{1}{4}\left[1+\ln \left(\frac{8 \mu^{2} \xi^{2}}{\nu^{4}}\right)\right] \text {, }
$$

the curvaton will start oscillating in the minimum of its potential. During the tracking phase $r(x) \simeq r_{\text {tr }}=4 \mu^{2} \ll 1$. During the oscillatory regime the energy density of the curvaton will decrease as $a^{-3}$ so that

$$
r(x)=4 \mu^{2}\left(\frac{a}{a_{\mathrm{m}}}\right), \quad x \geqslant x_{\mathrm{m}} .
$$

Let us now write, as a first step, the evolution equations for the fluctuations in the case when the background after the end of inflation is suddenly dominated by radiation:

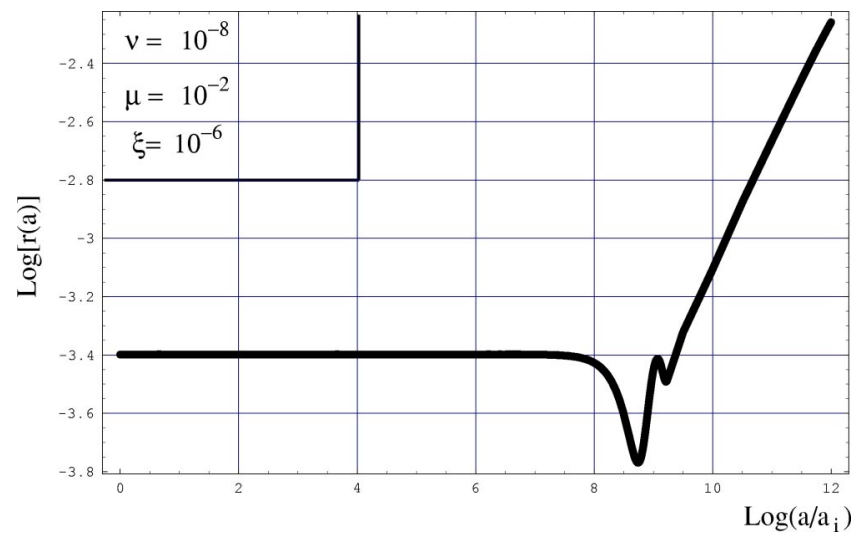

FIG. 1. The numerical results for the evolution of $r$ [see Eq. (3.7)] are reported.

$$
\begin{gathered}
\frac{d \Phi_{k}}{d x}+\Phi_{k}=-\frac{\delta_{\mathrm{r}}(k)}{2}-\frac{1}{6}\left[-\Phi_{k}\left(\frac{d \boldsymbol{\epsilon}}{d x}\right)^{2}+\frac{d \tilde{\epsilon}}{d x} \frac{d \tilde{\chi}_{k}}{d x}\right. \\
\left.+e^{4 x} \frac{\nu^{4}}{\mu \xi^{2}} \sinh (\tilde{\epsilon} / \mu) \tilde{\chi}_{k}\right], \\
\frac{d^{2} \Phi_{k}}{d x^{2}}+2 \frac{d \Phi_{k}}{d x}-\Phi_{k}=\frac{\delta_{\mathrm{r}}(k)}{2}-\frac{1}{2}\left[\Phi_{k}\left(\frac{d \tilde{\boldsymbol{\epsilon}}}{d x}\right)^{2}-\frac{d \tilde{\epsilon}}{d x} \frac{d \tilde{\chi}_{k}}{d x}\right. \\
\left.+e^{4 x} \frac{\nu^{4}}{\mu \xi^{2}} \sinh (\tilde{\boldsymbol{\epsilon}} / \mu) \tilde{\chi} k\right] \\
\frac{d^{2} \tilde{\chi}_{k}}{d x^{2}}+\frac{d \tilde{\chi}_{k}}{d x}+e^{4 x} \frac{\nu^{4}}{\mu^{2} \xi^{2}} \cosh (\tilde{\boldsymbol{\epsilon}} / \mu) \tilde{\chi} \tilde{\chi}_{k}-4 \frac{d \tilde{\epsilon}}{d x} \frac{d \Phi_{k}}{d x} \\
+2 \frac{\nu^{4}}{\mu \xi^{2}} e^{4 x} \sinh (\tilde{\epsilon} / \mu) \Phi_{k}=0, \\
\frac{d x}{d x}\left[\delta_{\mathrm{r}}(k)-4 \Phi_{k}\right]=0 .
\end{gathered}
$$

Combining Eqs. (2.23) and (2.24)

$$
\begin{aligned}
& \frac{d^{2} \Phi_{k}}{d x^{2}}+3 \frac{d \Phi_{k}}{d x} \\
& +\frac{1}{3}\left[\Phi_{k}\left(\frac{d \tilde{\epsilon}}{d x}\right)^{2}-\frac{d \tilde{\epsilon}}{d x} \frac{d \chi_{k}}{d x}+2 e^{4 x} \frac{\nu^{4}}{\mu \xi^{2}} \sinh (\tilde{\epsilon} / \mu) \tilde{\chi}_{k}\right]=0 .
\end{aligned}
$$

The evolution equations of $\epsilon$ and of the fluctuations can be numerically integrated. Analytical approximations can also be obtained. In Fig. 1 the numerical results are illustrated, for a typical set of parameters, in terms of $r(x)$ as defined in Eq. (3.7). It can be appreciated that after a phase where $r^{\prime} \simeq 0$, the energy density of the $\epsilon$ will decrease, implying that $r(x) \sim e^{x}$. In Fig. 2 the evolution of $\epsilon(x)$ is reported. The 
numerical results (full line) are compared with the analytical approximation (dashed line). In fact, a useful analytical approximation to the whole evolution can be obtained by matching the solution (3.8), valid during the tracking regime, with the exact solution (of the approximate potential) valid in the oscillating regime. In order to do so it is useful to write the evolution equation for $\tilde{\epsilon}$ as

$$
\frac{d^{2} \tilde{\epsilon}}{d y^{2}}+\frac{3}{2 y} \frac{d \tilde{\epsilon}}{d y}+\tilde{\epsilon}=0, \quad y=e^{2 x} \frac{\nu^{2}}{2 \mu \xi},
$$

whose solution is

$$
\tilde{\boldsymbol{\epsilon}}(x)=e^{-x / 2}\left[A J_{1 / 4}\left(e^{2 x} \frac{\nu^{2}}{2 \mu \xi}\right)+B Y_{1 / 4}\left(e^{2 x} \frac{\nu^{2}}{2 \mu \xi}\right)\right], \quad x>x_{\mathrm{m}},
$$

where the numerical constants

$$
\begin{aligned}
& A=\frac{e^{x_{\mathrm{m}} / 2} \pi\left\{-4 \mu \xi Y_{1 / 4}\left(\frac{e^{2 x_{\mathrm{m}}} \nu^{2}}{2 \mu \xi}\right)-e^{2 x_{\mathrm{m}}} \nu^{2} Y_{5 / 4}\left(\frac{e^{2 x_{\mathrm{m}}} \nu^{2}}{2 \mu \xi}\right)\left[4 x_{\mathrm{m}}-\log \left(\frac{8 \mu^{2} \xi^{2}}{\nu^{4}}\right)\right]\right\}}{4 \xi}, \\
& B=\frac{e^{x_{\mathrm{m}} / 2} \pi\left\{4 \mu \xi J_{1 / 4}\left(\frac{e^{2 x_{\mathrm{m}}} \nu^{2}}{2 \mu \xi}\right)+e^{2 x_{\mathrm{m}}} \nu^{2} J_{5 / 4}\left(\frac{e^{2 x_{\mathrm{m}}} \nu^{2}}{2 \mu \xi}\right)\left[4 x_{\mathrm{m}}-\log \left(\frac{8 \mu^{2} \xi^{2}}{\nu^{4}}\right)\right]\right\}}{4 \xi},
\end{aligned}
$$

have been obtained by continuous matching of the solutions in $x_{\mathrm{m}}$. In more explicit terms

$$
\begin{aligned}
A & =-\pi e^{1 / 8} 2^{-5 / 8}\left[2 Y_{1 / 4}(\sqrt{2 e})+\sqrt{2 e} Y_{5 / 4}(\sqrt{2 e})\right] \nu^{-1 / 2} \mu^{5 / 4} \xi^{1 / 4} \\
& =-1.70146 \times \nu^{-1 / 2} \mu^{5 / 4} \xi^{1 / 4} \\
B & =\pi e^{1 / 8} 2^{-5 / 8}\left[2 J_{1 / 4}(\sqrt{2 e})+\sqrt{2 e} J_{5 / 4}(\sqrt{2 e})\right] \nu^{-1 / 2} \mu^{5 / 4} \xi^{1 / 4} \\
& =3.98472 \times \nu^{-1 / 2} \mu^{5 / 4} \xi^{1 / 4} .
\end{aligned}
$$

According to Fig. 2, the analytical approximation, based on the continuity of the solutions of Eqs. (3.8) and (3.17) compares very well with the numerical calculation.

Using the results for the evolution of $\epsilon(x)$ the amount of the fluctuations produced during the oscillating phase can be estimated. In Figs. 3 and 4, the numerical evolution for $\Phi_{k}$

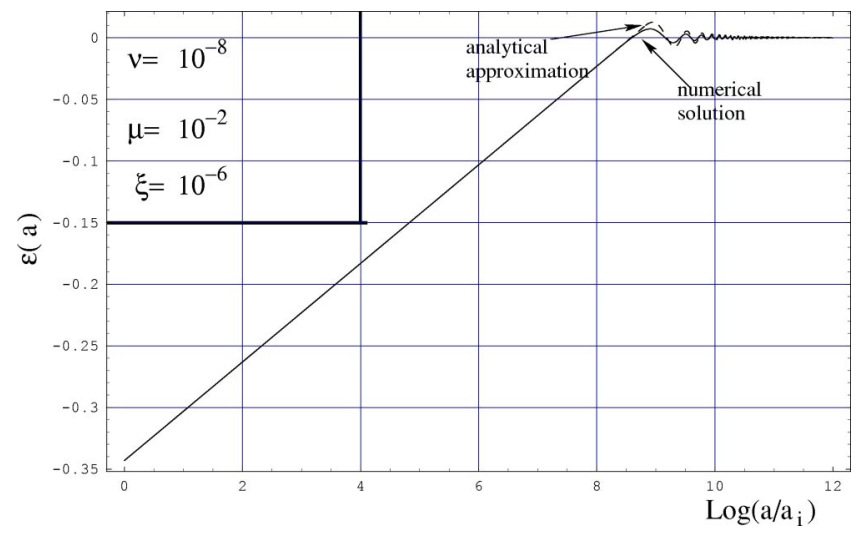

FIG. 2. The full line illustrates the numerical result while the dashed line shows the analytical approximation based on Eq. (3.17). and $\mathcal{R}_{k}$ are reported. After a flat plateau corresponding to the phase where $r(x)$ is constant, the adiabatic fluctuations grow. However, the final value of the adiabatic fluctuations (computed, for instance, at the moment when $\epsilon$ decays) will always be very small. In the integrations reported in Figs. 3 and 4 initial conditions are set according to Eqs. (2.27). In order to understand the smallness of the final value of the adiabatic fluctuations, recall that the asymptotic solution of the perturbation equations during the tracking can be written as

$$
\Phi_{k}^{(\mathrm{m})}=-\frac{2}{9} \mu \tilde{\chi}_{k}^{(i)}, \quad \chi_{k}^{(\mathrm{m})}=-\frac{4}{9} \mu^{2} \tilde{\chi}_{k}^{(\mathrm{i})},
$$

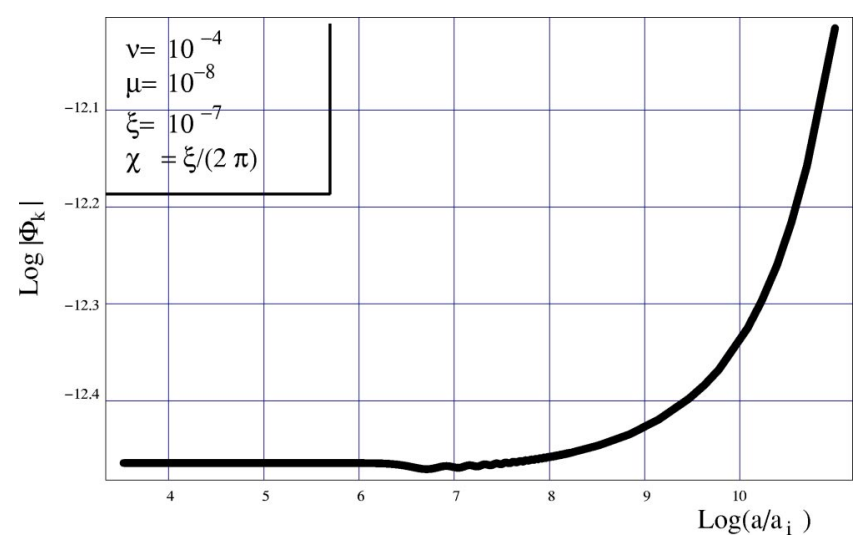

FIG. 3. The numerical evolution of $\Phi_{k}$ illustrated in the case where $M=10^{-4} M_{\mathrm{P}}, \Lambda=10^{-8} M_{\mathrm{P}}$ and $H_{i}=10^{-3} M$. Notice that the constant value of $\Phi_{k}$ is correctly reproduced by the analytical estimate given in Eq. (3.20). 


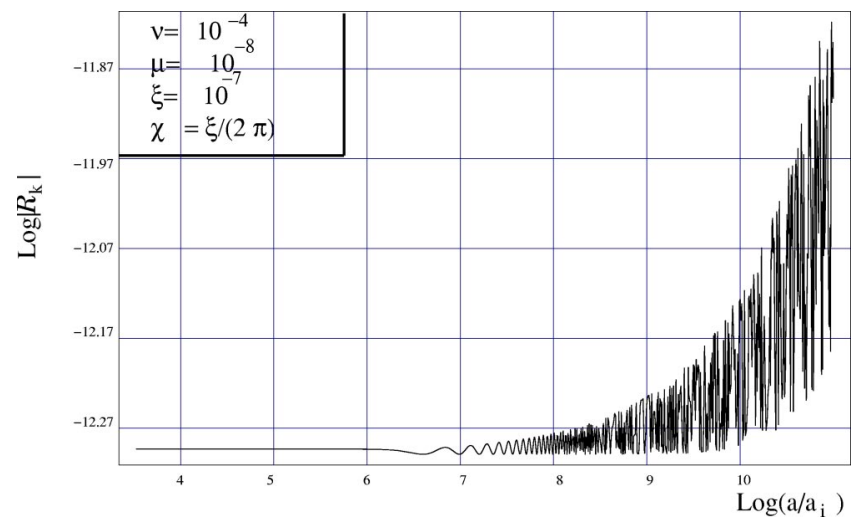

FIG. 4. The numerical evolution of $\mathcal{R}_{k}$ is reported for the same set of parameters discussed in Fig. 4.

implying that

$$
\mathcal{R}_{k}^{(\mathrm{m})}=-\frac{3}{2} \Phi_{k}-\frac{1}{2} \frac{d \Phi_{k}}{d x}=\frac{1}{3} \mu \tilde{\chi}_{k}^{(i)}
$$

The values obtained in Eqs. (3.20) and (3.21) are in excellent agreement with the amplitude of the flat plateau occurring prior to $x_{\mathrm{m}}$ (which is about 6.5 for the parameters chosen in Figs. 3 and 4). Different choice of parameters lead to the same qualitative features in the evolution of the fluctuations.

During the oscillating regime

$$
\frac{d \mathcal{R}_{k}}{d x} \simeq \frac{\partial W}{\partial \epsilon} \frac{\chi_{k}}{\rho_{\mathrm{r}}}, \quad r(x)=4 \mu^{2} e^{\left(x-x_{\mathrm{m}}\right)}, \quad x>x_{\mathrm{m}} .
$$

These solutions hold down to the moment of decay occurring at a typical curvature scale

$$
H_{\mathrm{d}} \sim\left(\frac{M^{2}}{\Lambda}\right)^{3} M_{\mathrm{P}}^{-2}
$$

where it has been taken into account that the effective mass is, during the oscillating phase $M^{2} / \Lambda$. Integrating the evolution equation for $\mathcal{R}_{k}$ it can indeed be obtained using Eqs. (3.20)-(3.22)

$$
\mathcal{R}_{k}^{(\mathrm{d})}=\mathcal{R}_{k}^{(\mathrm{m})}+2 \frac{r_{\mathrm{d}}}{\mu} \chi_{k}^{(\mathrm{m})} \simeq \frac{\mu}{3}\left(1-\frac{8}{3} r_{\mathrm{d}}\right) \tilde{\chi}_{k}^{(\mathrm{i})},
$$

where $r_{\mathrm{d}}=r\left(x_{\mathrm{d}}\right)$ and $x_{\mathrm{d}}$ is determined through Eq. (3.23). Since $\mu \ll 1$ (to ensure that $\epsilon$ does not dominate already during the tracking phase) and since $r_{\mathrm{d}} \leqslant 1$, Eq. (3.24) implies that the final value $\mathcal{R}_{k}^{(\mathrm{d})}$ will be much smaller than $\tilde{\chi}_{k}^{(\mathrm{i})}$ $\sim \xi /(2 \pi)$.

\section{MINIMAL INFLATIONARY SCALE}

In the standard curvaton scenario the energy density of the curvaton increases with time with respect to the energy density of the radiation background. From this aspect of the theoretical construction, a number of constraints can be derived; these include an important aspect of the inflationary dynamics occurring prior to the curvaton oscillations, namely the minimal curvature scale at the end of inflation compatible with the curvaton idea. It has been shown, in the previous sections, that to have a phase of tracking curvaton, unfortunately, does not help. In the present section the bounds on the inflationary curvature scale will be reviewed, with particular attention to the case where the postinflationary phase is not suddenly dominated by radiation like in the case of quintessential inflation $[17,9]$.

\section{A. The standard argument}

First the standard argument will be reviewed (see [14] for a particularly lucid approach to this problem). Suppose, for simplicity, that the curvaton field $\epsilon$ has a massive potential and that its evolution, after the end of inflation, occurs during a radiation dominated stage of expansion. The field $\epsilon$ starts oscillating at a typical scale $H_{\mathrm{m}} \sim m$ and the ratio between the curvaton energy density and the energy density of the radiation background is, roughly,

$$
r(t) \simeq\left(\frac{\epsilon_{\mathrm{i}}}{M_{\mathrm{P}}}\right)^{2}\left(\frac{a}{a_{\mathrm{m}}}\right), \quad H<H_{\mathrm{m}} .
$$

When $\epsilon$ decays the ratio $r$ gets frozen to its value at decay, i.e. $r(t) \simeq r\left(t_{\mathrm{d}}\right)=r_{\mathrm{d}}$ for $t>t_{\mathrm{d}}$. Equation (4.1) then implies

$$
m=\frac{\epsilon_{\mathrm{i}}^{2}}{r_{\mathrm{d}} M_{\mathrm{P}}} \text {. }
$$

The energy density of the background fluid just before decay has to be larger than the energy density of the decay products, i.e. $\rho_{\mathrm{r}}\left(t_{\mathrm{d}}\right) \geqslant T_{\mathrm{d}}^{4}$. Since

$$
\rho_{\mathrm{r}}\left(t_{\mathrm{d}}\right) \simeq m^{2} M_{\mathrm{P}}^{2}\left(\frac{a_{\mathrm{m}}}{a_{\mathrm{d}}}\right)^{4},
$$

the mentioned condition implies

$$
\frac{m}{T_{\mathrm{d}}} \sqrt{\frac{m}{M_{\mathrm{P}}}}>1,
$$

which can also be written, using Eq. (4.2), as

$$
\left(\frac{\epsilon_{\mathrm{i}}}{M_{\mathrm{P}}}\right)^{3} \geqslant r_{\mathrm{d}}^{3 / 2}\left(\frac{T_{\mathrm{d}}}{M_{\mathrm{P}}}\right) \text {. }
$$

Equation (4.5) has to be compared with the restrictions coming from the amplitude of the adiabatic perturbations, which should be consistent with observations. If $\epsilon$ decays before becoming dominant the curvature perturbations at the time of decay are

$$
\left.\mathcal{R}_{k}\left(t_{\mathrm{d}}\right) \simeq \frac{1}{\rho_{\mathrm{r}}} \frac{\partial W}{\partial \epsilon} \chi\right|_{t_{\mathrm{d}}} \simeq r_{\mathrm{d}} \frac{\chi_{k}^{\mathrm{i}}}{\epsilon_{\mathrm{i}}} .
$$

Recalling that $\chi_{k}^{\mathrm{i}} \sim H_{\mathrm{i}} /(2 \pi)$ the power spectrum of curvature perturbations 


$$
\mathcal{P}^{1 / 2} \simeq \frac{r_{\mathrm{d}} H_{\mathrm{i}}}{4 \pi \epsilon_{\mathrm{i}}} \simeq 5 \times 10^{-5}
$$

implies, using Eq. (4.5) together with Eq. (4.2),

$$
\left(\frac{H_{\mathrm{i}}}{M_{\mathrm{P}}}\right) \geqslant 10^{-4} \times r_{\mathrm{d}}^{-1 / 2}\left(\frac{T_{\mathrm{d}}}{M_{\mathrm{P}}}\right)^{1 / 3} .
$$

Recalling now that $r_{\mathrm{d}} \leqslant 1$ and $m<H_{\mathrm{i}}$, the above inequality implies that, at most, $H_{\mathrm{i}} \geqslant 10^{-12} M_{\mathrm{P}}$ if $T_{\mathrm{d}} \sim 1 \mathrm{MeV}$ is selected. This estimate is, in a sense, general since the specific relation between $T_{\mathrm{d}}$ and $H_{\mathrm{d}}$ is not fixed. The bound (4.8) can be even more constraining, for certain regions of parameter space, if the condition $T_{\mathrm{d}} \geqslant \sqrt{H_{\mathrm{d}} M_{\mathrm{P}}}$ is imposed with $H_{\mathrm{d}}$ $\simeq m^{3} / M_{\mathrm{P}}^{2}$. In this case, Eq. (4.8) implies $H_{\mathrm{i}}^{2} \geqslant 10^{-8} m M_{\mathrm{P}}$, which is more constraining than the previous bound for sufficiently large values of the mass, i.e. $m \geqslant 10^{-4} H_{\mathrm{i}}$. Thus, in the present context, the inflationary curvature scale is bound to be in the interval $10^{-12} M_{\mathrm{P}} \leqslant H_{\mathrm{i}} \leqslant 10^{-6} M_{\mathrm{P}}$.

\section{B. Different post-inflationary histories}

Consider now the case where the inflationary epoch is not immediately followed by radiation. Different models of this kind may be constructed. For instance, if the inflaton field is identified with the quintessence field, a long kinetic phase occurred prior to the usual radiation-dominated stage of expansion [17] (see also [23] for some other references not directly related to the present calculation). The evolution of a massive curvaton field in quintessential inflationary models has been recently studied [9] in the simplest scenario where the curvaton field is decoupled from the quintessence field and it is minimally coupled to the metric. In order to be specific, suppose that, as in [17], the inflaton potential, $V(\varphi)$ is chosen to be a typical power law during inflation and an inverse power during the quintessential regime:

$$
\begin{aligned}
& V(\varphi)=\lambda\left(\varphi^{4}+M^{4}\right), \quad \varphi<0, \\
& V(\varphi)=\frac{\lambda M^{8}}{\varphi^{4}+M^{4}}, \quad \varphi \geqslant 0,
\end{aligned}
$$

where $\lambda$ is the inflaton self-coupling and $M$ is the typical scale of quintessential evolution. The potential of the curvaton may be taken to be, for simplicity, quadratic. In this model the curvaton will evolve, right after the end of inflation, in an environment dominated by the kinetic energy of $\varphi$. The curvaton starts oscillating at $H_{\mathrm{m}} \sim m$ and becomes dominant at a typical curvature scale $H_{\epsilon} \sim m\left(\epsilon_{\mathrm{i}} / M_{\mathrm{P}}\right)^{2}$. Due to the different evolution of the background geometry, the ratio $r(t)$ will take the form

$$
r(t) \simeq m^{2}\left(\frac{\epsilon_{\mathrm{i}}}{M_{\mathrm{P}}}\right)^{2}\left(\frac{a}{a_{\mathrm{m}}}\right)^{3}, \quad H<H_{\mathrm{m}}
$$

to be compared with Eq. (4.1) valid in the standard case. For $t>t_{\mathrm{d}}, r(t)$ gets frozen to the value $r_{\mathrm{d}}$ whose relation to $\epsilon_{\mathrm{i}}$ is different from the one obtained previously [see Eq. (4.2)] and valid in the case when $\epsilon$ relaxes in a radiation dominated environment. In fact, from Eq. (4.10),

$$
m \simeq \frac{\epsilon_{\mathrm{i}}}{\sqrt{r_{\mathrm{d}}}}
$$

From the requirement

$$
\rho_{\mathrm{k}}\left(t_{\mathrm{d}}\right) \simeq m^{2} M_{\mathrm{P}}^{2}\left(\frac{a_{\mathrm{m}}}{a_{\mathrm{d}}}\right)^{6} \geqslant T_{\mathrm{d}}^{4},
$$

it can be inferred, using Eq. (4.11), that

$$
\left(\frac{\epsilon_{\mathrm{i}}}{M_{\mathrm{P}}}\right)^{3 / 2} \geqslant r_{\mathrm{d}}^{3 / 4}\left(\frac{T_{\mathrm{d}}}{M_{\mathrm{P}}}\right) \text {. }
$$

Following the analysis reported in [9], the amount of produced fluctuations can be computed. The spatial curvature perturbation can be written, in this model, as

$$
\mathcal{R}_{k}=-\frac{H}{\dot{\varphi}^{2}+\dot{\epsilon}^{2}}\left[\dot{\varphi} v_{\varphi}+\dot{\epsilon} v_{\epsilon}\right]
$$

where

$$
\begin{gathered}
v_{\varphi}=\chi_{\varphi}+\frac{\dot{\varphi}}{H} \Phi, \\
v_{\epsilon}=\chi_{\epsilon}+\frac{\dot{\epsilon}}{H} \Phi,
\end{gathered}
$$

are, respectively, the canonically normalized fluctuations of $\varphi$ and $\epsilon$. As discussed in [9] the relevant evolution equations can be written as

$$
\begin{gathered}
\ddot{v}_{\varphi}+3 H \dot{v}_{\varphi}-\frac{1}{a^{2}} \nabla^{2} v_{\varphi}+\left[\frac{\partial^{2} V}{\partial \varphi^{2}}-\frac{1}{M_{\mathrm{P}}^{2} a^{3}} \frac{\partial}{\partial t}\left(\frac{a^{3}}{H} \dot{\varphi}^{2}\right)\right] v_{\varphi} \\
-\frac{1}{M_{\mathrm{P}}^{2} a^{3}} \frac{\partial}{\partial t}\left(\frac{a^{3}}{H} \dot{\varphi} \dot{\epsilon}\right) v_{\epsilon}=0, \\
\ddot{v}_{\epsilon}+3 H \dot{v}_{\epsilon}-\frac{1}{a^{2}} \nabla^{2} v_{\epsilon}+\left[\frac{\partial^{2} W}{\partial \epsilon^{2}}-\frac{1}{M_{\mathrm{P}}^{2} a^{3}} \frac{\partial}{\partial t}\left(\frac{a^{3}}{H} \dot{\epsilon}^{2}\right)\right] v_{\epsilon} \\
-\frac{1}{M_{\mathrm{P}}^{2} a^{3}} \frac{\partial}{\partial t}\left(\frac{a^{3}}{H} \dot{\varphi} \dot{\epsilon}\right) v_{\varphi}=0 .
\end{gathered}
$$

Solving Eqs. (4.17) and (4.18) with the appropriate initial conditions, and using that $a^{3} \dot{\varphi}^{2} / H$ is constant during the kinetic phase, Eq. (4.14) can be written as [9]

$$
\mathcal{R}_{k}(t) \simeq \frac{H}{\rho_{\mathrm{k}}} \dot{\epsilon} v_{\epsilon} \simeq \frac{\chi_{\epsilon}}{\rho_{\mathrm{k}}} \frac{\partial W}{\partial \epsilon} \simeq r_{\mathrm{d}}\left(\frac{\chi_{k}^{(\mathrm{i})}}{\epsilon_{\mathrm{i}}}\right) .
$$

Recalling that $\chi_{k}^{(\mathrm{i})} \sim H_{\mathrm{i}} /(2 \pi)$, the observed value of the power spectrum, i.e. $\mathcal{P}_{\mathcal{R}}^{1 / 2} \sim 5 \times 10^{-5}$, implies 


$$
\left(\frac{H_{\mathrm{i}}}{M_{\mathrm{P}}}\right) \geqslant 10^{-4}\left(\frac{T_{\mathrm{d}}}{M_{\mathrm{P}}}\right)^{2 / 3} .
$$

The same approximations discussed in the standard case will now be applied to the case of quintessential inflation. Suppose that $T_{\mathrm{d}} \sim 1 \mathrm{MeV}$ (i.e. the minimal value compatible with nucleosynthesis considerations). Thus, from Eq. (4.20) $H_{\mathrm{i}} \geqslant 10^{-19} M_{\mathrm{P}}$. This estimate has to be compared with $H_{\mathrm{i}}$ $\geqslant 10^{-12} M_{\mathrm{P}}$ which was obtained [see Eq. (4.8)] in the case when $\epsilon$ relaxes to its minimum in a radiation dominated environment. Suppose now that the decay of $\epsilon$ is purely gravitational, i.e. $\Gamma \sim m^{3} / M_{\mathrm{P}}$. If this is the case, as previously argued, one could also require that $T_{\mathrm{d}} \geqslant \sqrt{H_{\mathrm{d}} M_{\mathrm{P}}}$. This requirement implies that for masses $m>10 \mathrm{TeV}, H_{\mathrm{i}}$ $>10^{-4} \mathrm{~m}$.

\section{CONCLUDING REMARKS}

In the present investigation the possibility that the ratio between the curvaton energy density and that of the dominant component of the background sources is constant during a significant part in the evolution of the Universe. The vari- ous estimates of this preliminary analysis seem to suggest that if $r^{\prime} \simeq 0$ for a sufficiently long time the obtained curvature perturbations present prior to curvaton decay are much smaller than the value required by observations. The possibility of having $r^{\prime} \simeq 0$ down to sufficiently low curvature scales would, on the other hand, be interesting to relax the bounds on the minimal inflationary curvature scale, which, in the standard scenario (where $r^{\prime}>0$ ), is roughly $H_{\mathrm{i}}$ $>10^{-12} M_{\mathrm{P}}$ for the most optimistic set of parameters. If this bound could be evaded inflation could take place, within the framework discussed in the present paper, also at much smaller curvature scales. In this sense, the presence of a phase $r^{\prime}=0$ does not alleviate the problem. Furthermore, if $r^{\prime}=0$ for a sufficiently long time, the final amount of curvature perturbations gets drastically reduced.

In a related perspective the case of low-scale quintessential inflation has been examined. In this case, $r^{\prime}>0$ and the background geometry is kinetically dominated down to the moment of curvaton oscillations. The same argument, leading to the standard bound on the minimal inflationary curvature scale shows, in this case, that the bounds are a bit relaxed and curvature scales $H_{\mathrm{i}}>10^{-19} M_{\mathrm{P}}$ become plausible.
[1] D. Lyth and D. Wands, Phys. Lett. B 524, 5 (2002).

[2] T. Moroi and T. Takahashi, Phys. Rev. D 66, 063501 (2002).

[3] K. Enqvist and M. Sloth, Nucl. Phys. B626, 395 (2002).

[4] M. Sloth, Nucl. Phys. B656, 239 (2003).

[5] V. Bozza et al., Phys. Lett. B 543, 14 (2002); 67, 063514 (2003).

[6] S. Mollerach, Phys. Rev. D 42, 313 (1990).

[7] N. Bartolo and A.R. Liddle, Phys. Rev. D 65, 121301 (2002).

[8] D.H. Lyth, C. Ungarelli, and D. Wands, Phys. Rev. D 67, 023503 (2003).

[9] M. Giovannini, Phys. Rev. D 67, 123512 (2003).

[10] M. Bastero-Gil, V. Di Clemente, and S.F. King, Phys. Rev. D 67, 083504 (2003); 67, 103516 (2003).

[11] K. Enqvist, A. Jokinen, S. Kasuya, and A. Mazumdar, Phys. Rev. D 68, 103507 (2003).

[12] K. Enqvist, S. Kasuya, and A. Mazumdar, Phys. Rev. Lett. 90, 091302 (2003).

[13] K. Dimopoulos et al., J. High Energy Phys. 05, 057 (2003).
[14] D. Lyth, Phys. Lett. B 579, 239 (2004).

[15] K. Dimopoulos, Phys. Rev. D 68, 123506 (2003).

[16] M. Fujii and T. Yanagida, Phys. Rev. D 66, 123515 (2002).

[17] P.J.E. Peebles and A. Vilenkin, Phys. Rev. D 59, 063505 (1999).

[18] M. Giovannini, Phys. Rev. D 60, 123511 (1999); Class. Quantum Grav. 16, 2905 (1999).

[19] T. Barreiro, E.J. Copeland, and N.J. Nunes, Phys. Rev. D 61, 127301 (2000).

[20] A. Albrecht and C. Skordis, Phys. Rev. Lett. 84, 2076 (2000).

[21] V. Sahni and L. Wang, Phys. Rev. D 62, 103517 (2000).

[22] P.J. Peebles and B. Ratra, Rev. Mod. Phys. 75, 559 (2003).

[23] L. Ford, Phys. Rev. D 35, 2955 (1987); B. Spokoiny, Phys. Lett. B 315, 40 (1993); R.A. Frewin and J.E. Lidsey, Int. J. Mod. Phys. D 2, 323 (1993); P. Ferreira and M. Joyce, Phys. Rev. D 58, 023503 (1998); M. Joyce and T. Prokopec, ibid. 57, 6022 (1998). 